\title{
Code and Solution Verification Assessment of the CTF Thermal Hydraulic Subchannel Code
}

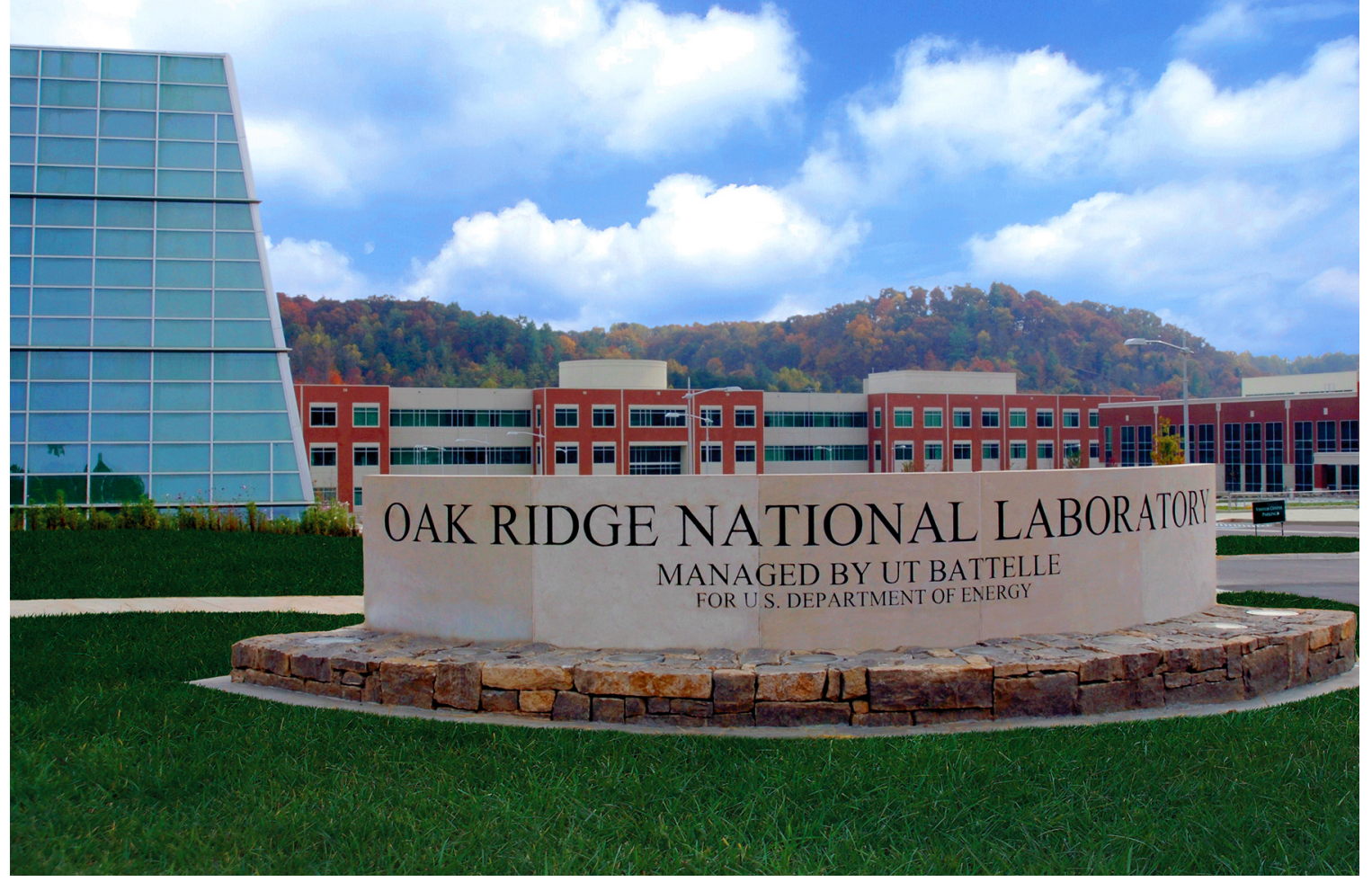
R. Salko*
M. Pilch
V. Kumar*

September 30, 2021

Approved for public release.

Distribution is unlimited. 


\section{DOCUMENT AVAILABILITY}

Reports produced after January 1, 1996, are generally available free via US Department of Energy (DOE) SciTech Connect.

Website: www.osti.gov/

Reports produced before January 1, 1996, may be purchased by members of the public from the following source:

National Technical Information Service

5285 Port Royal Road

Springfield, VA 22161

Telephone: 703-605-6000 (1-800-553-6847)

TDD: $703-487-4639$

Fax: 703-605-6900

E-mail: info@ntis.gov

Website: http://classic.ntis.gov/

Reports are available to DOE employees, DOE contractors, Energy Technology Data Exchange representatives, and International Nuclear Information System representatives from the following source:

Office of Scientific and Technical Information

PO Box 62

Oak Ridge, TN 37831

Telephone: 865-576-8401

Fax: 865-576-5728

E-mail: report@osti.gov

Website: http://www.osti.gov

This report was prepared as an account of work sponsored by an agency of the United States Government. Neither the United States Government nor any agency thereof, nor any of their employees, makes any warranty, express or implied, or assumes any legal liability or responsibility for the accuracy, completeness, or usefulness of any information, apparatus, product, or process disclosed, or represents that its use would not infringe privately owned rights. Reference herein to any specific commercial product, process, or service by trade name, trademark, manufacturer, or otherwise, does not necessarily constitute or imply its endorsement, recommendation, or favoring by the United States Government or any agency thereof. The views and opinions of authors expressed herein do not necessarily state or reflect those of the United States Government or any agency thereof. 
ORNL/TM-2021/2203

Nuclear Energy and Fuel Cycle Division

\title{
Code and Solution Verification Assessment of the CTF Thermal Hydraulic Subchannel Code
}

\author{
R. Salko*, M. Pilch ${ }^{\dagger}$, V. Kumar* \\ * Oak Ridge National Laboratory, Oak Ridge, TN \\ $\dagger$ MPilchConsulting, Tijeras, NM
}

Date Published: September 30, 2021

Prepared by

OAK RIDGE NATIONAL LABORATORY

Oak Ridge, TN 37831-6283

managed by

UT-Battelle LLC

for the

US DEPARTMENT OF ENERGY

under contract DE-AC05-00OR22725 



\section{CONTENTS}

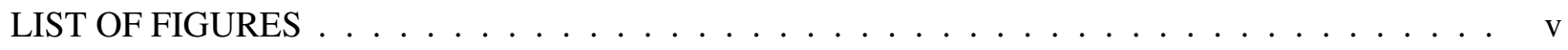

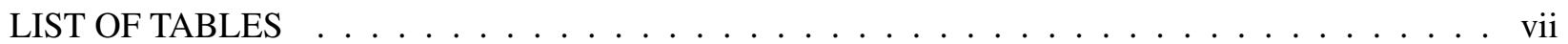

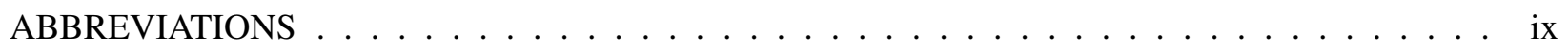

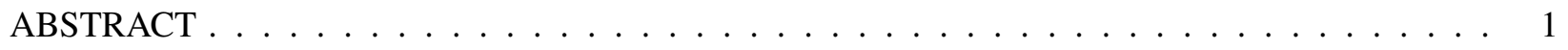

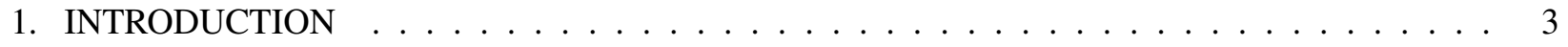

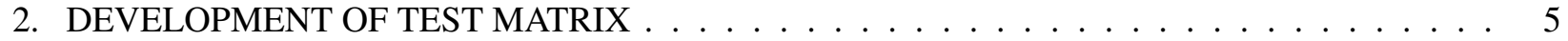

3. GRID SPACER ENHANCEMENT TEST $\ldots \ldots \ldots \ldots \ldots \ldots \ldots \ldots$

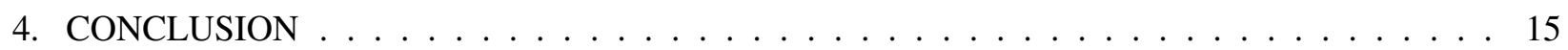

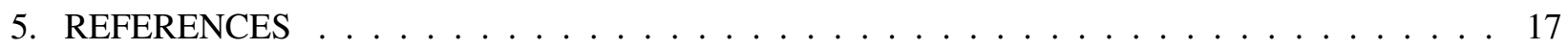





\section{LIST OF FIGURES}

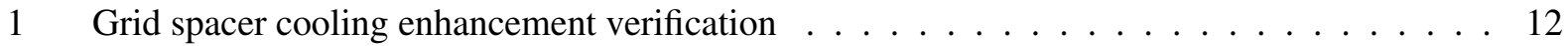

2 Grid spacer shear enhancement verification . . . . . . . . . . . . . . . . 13

3 TKE convergence rate with level defect. . . . . . . . . . . . . . . . . . . 14 



\section{LIST OF TABLES}

$1 \mathrm{CTF}$ code and solution verification matrix $\ldots \ldots \ldots \ldots$ 



\section{ABBREVIATIONS}

BEPU Best Estimate Plus Uncertainty

CASL Consortium for Advanced Simulation of Light Water Reactors

CHF critical heat flux

CILC crud-induced localized corrosion

CIPS crud-induced power shift

CSAU Code Scaling, Applicability, and Uncertainty

DNB departure from nucleate boiling

EMDAP Evaluation Model Development and Assessment Process

LBLOCA large-break loss-of-coolant accident

LHS left-hand side

LOCA loss-of-coolant accident

NPP nuclear power plant

PWR pressurized water reactor

RHS right-hand side

RIA reactivity insertion accident

SQA software quality assurance

TKE turbulent kinetic energy

VERA Virtual Environment for Reactor Applications

YHL Yao-Hochreiter-Leech 



\begin{abstract}
CTF is a thermal-hydraulics subchannel code jointly developed by Oak Ridge National Laboratory and North Carolina State University. Over the past seven years, the Consortium for Advanced Simulation of Light Water Reactors (CASL) has made a significant investment in developing CTF so it can be used to model light water reactors, including nominal operating conditions, departure from nucleate boiling analysis, and transients ranging from loss of flow to reactivity insertion accidents. In addition to implementing new modeling capabilities and developing the user input and output interface, extensive work has been performed to improve the code's quality assurance program, resulting in a development process that conforms with NQA-1 requirements. The CASL program follows the Predictive Capability Maturity Model (PCMM) approach for assessing code quality, which emphasizes performing code verification (ensuring the code converges to the correct answer) and solution verification (ensuring the code converges for the intended application). Code and solution verification are used to identify uncertainty errors introduced by numerical approximations in the code and are important for demonstrating that the model is coded without error, which is an important aspect of the Best Estimate plus Uncertainty method. This paper presents a comprehensive overview of the code and solution verification testing that has been performed on CTF. A top-down approach is taken in which the intended CTF applications are presented, followed by the code features required for their modeling. These features are then linked to the applicable code and solution verification tests that demonstrate proper functioning. Past testing efforts are summarized, and new tests are added to help close gaps in the presented test matrix. Rather than performing "one-off" exercises, these tests are added to the automated CTF regression test suite to ensure continual code quality.
\end{abstract}





\section{INTRODUCTION}

Best Estimate Plus Uncertainty (BEPU) finds acceptance in the regulatory arena because it provides a rigorous framework for quantifying uncertainties and scaling them to address nuclear power plant (NPP) issues at full scale. BEPU necessarily provides focus on the controlling physics and assures that codes have the necessary capabilities. Early applications of BEPU (Code Scaling, Applicability, and Uncertainty (CSAU) and Evaluation Model Development and Assessment Process (EMDAP), see Rohatgi and Kaizer [2020]) did not explicitly require evidence that code capabilities were implemented correctly (i.e., that they were free of defects). In the arena of modern engineering codes, we now require evidence that codes be developed and maintained according to accepted standards of software quality assurance (SQA). Formal software testing, usually in the form of regression testing, is integral to those SQA processes.

Early applications of BEPU focused on large-break loss-of-coolant accident (LBLOCA), in which the notion of "nodalization" was associated with control volumes and most physics was represented through constitutive models. Nodalization was considered part of the model, and iteration was explicitly allowed to optimize comparisons with experiment data. There is no expectation that nodalization can be refined arbitrarily to obtain a converged solution for these codes. Because iteration means calibration, the process requires that the same nodalization be carried forward to NPP calculations to ensure that differences in nodalization do not introduce unwanted distortions in NPP simulations. The application of BEPU has now extended to issues beyond LBLOCA. Much of the physics may be represented in the form of differential equations which are solved using finite difference, finite volume, or finite element solution schemes.

The notion of nodalization is replaced with the concept of discretization, which is also sometimes referred to as the "grid." It is wrong to consider the grid as part of the model; instead, the grid is foundational to the solution of the model. Calibration of the grid to optimize comparisons with separate or integral effects tests is unacceptable. The proper perspective is to seek evidence that the code will converge to the correct answer for the intended application as the grid is refined and to quantify discretization errors for any practical grid used in an application. This requires evidence of code and solution verification, which builds upon a foundation of quality SQA practices and an extensive body of unit and regression tests. This is explicit in some modern reformulations of BEPU (Unal et al. [2011]).

Code verification tests the code's ability to converge to the correct answer, which is in the form of a benchmark with a known analytic or manufactured solution. Solution verification tests the ability of a code to converge for an intended application where the correct answer is not known. Combining code and solution verification together allows inference that the code converges to the correct answer for the intended application. We strongly emphasize quantifying convergence behavior, both in code testing and applications. Comparing the computed order of convergence with expectations of the numerical scheme is a very effective way of detecting code bugs and algorithm deficiencies. The latter are poorly addressed with typical regression testing. Our systematic approach to code verification (with a focus on convergence behavior) has identified issues in the coding that were not previously recognized, even though CTF is a mature code with years of user experience. CTF (Salko et al. [2019a]), a two-fluid subchannel thermal hydraulics code derived from the legacy COBRA-TF code (Thurgood et al. [1983]), is developed and maintained with strict SQA practices (currently NQA-1 compliant), and it has an extensive regression test suite, with about $80 \%$ line coverage.

Transparency is another attribute that we emphasize. It is not enough to say a code is developed to recognized SQA practices and is heavily tested. It should also be transparent to users and regulatory 
agencies, so they can readily see what physics has been tested, what the tests are, and where gaps in testing might exist. This paper illustrates an approach to meet these needs using the CTF code as a recent case study. The physics-based test matrix is an effective way of communicating this information to users and regulatory agencies.

The assessment of numerical errors in a full-scale plant calculation through systematic grid refinement might not be practical given current computer resources. However, a building block approach can be used to infer grid adequacy at full scale. Quantified convergence behavior at the physics level guides selection of default grid sizes. Solution verification studies at the assembly level test the adequacy of default grids.

CTF has been jointly developed by Oak Ridge National Laboratory and North Carolina State University as part of the Consortium for Advanced Simulation of Light Water Reactors (CASL) program. CTF has been incorporated into the Virtual Environment for Reactor Applications (VERA) (Kochunas et al. [2017]), which is a high-fidelity multiphysics reactor core simulator developed by CASL. In VERA, CTF has been coupled to the neutronics code MPACT (Collins et al. [2016]) and the crud chemistry code MAMBA (Collins et al. [2017]). VERA has been developed to model pressurized water reactor (PWR) reactor depletions, departure from nucleate boiling (DNB), crud-induced power shift (CIPS), and reactivity insertion accidents (RIAs). 


\section{DEVELOPMENT OF TEST MATRIX}

A test matrix was developed to identify testing needs in CTF. A top-down approach is being applied in which the intended code applications are identified first, and then code modeling requirements that support those applications are identified. Requirements are identified only for models that will have an expected rate of convergence and that can be checked in the code verification problems being considered. Closure laws are algebraic expressions that do not exhibit convergence behavior in themselves, and quality assurance is best addressed with unit tests. This will be the subject of follow-on work.

Once code applications and modeling requirements are identified, tests are developed that will ensure the proper functioning of the modeling capability. In some cases, testing had already been performed in the past and simply needed to be mapped to the requirement. In other cases, gaps were identified in the testing matrix that needed to be closed by creating and performing new tests.

Whereas CTF is a two-phase code that was originally developed for loss-of-coolant accident (LOCA) and post-critical heat flux (CHF) modeling applications, its use in CASL has mostly been limited to single-phase PWR modeling applications, including steady-state prediction of normal operating conditions (reactor depletion), prediction of DNB, and prediction of crud growth and its effect on CIPS and crud-induced localized corrosion (CILC). These CASL applications form the basis for applications to be tested in this activity.

At the highest level, modeling of each application space requires a coupled set of governing equations to be solved, including mass, momentum, and energy (fluid and solid). The general forms of the four primary governing equations of interest are shown in Eqs. 1-4, which include conservation of mass, momentum, liquid energy, and solid energy, respectively.

$$
\begin{gathered}
\frac{\partial}{\partial t}\left(\alpha_{k} \rho_{k}\right)+\nabla \cdot\left(\alpha_{k} \rho_{k} \vec{V}_{k}\right)=L_{k}+M_{e}^{T} \\
\frac{\partial}{\partial t}\left(\alpha_{k} \rho_{k} \vec{V}_{k}\right)+\frac{\partial}{\partial x}\left(\alpha_{k} \rho_{k} u_{k} \vec{V}_{k}\right)+\frac{\partial}{\partial y}\left(\alpha_{k} \rho_{k} v_{k} \vec{V}_{k}\right)+\frac{\partial}{\partial z}\left(\alpha_{k} \rho_{k} w_{k} \vec{V}_{k}\right)= \\
\alpha_{k} \rho_{k} \vec{g}-\alpha_{k} \nabla P+\nabla \cdot \alpha_{k} \tau_{k}^{i j}+\vec{M}_{k}^{L}+\vec{M}_{k}^{d}+\vec{M}_{k}^{T} \\
\frac{\partial}{\partial t}\left(\alpha_{k} \rho_{k} h_{k}\right)+\nabla \cdot\left(\alpha_{k} \rho_{k} h_{k} \vec{V}_{k}\right)=-\nabla \cdot \alpha_{k} \vec{q}_{k}^{T}+\Gamma_{k} h_{k}^{i}+q_{w k}^{\prime \prime \prime}+\alpha_{k} \frac{\partial P}{\partial t} \\
\frac{d}{d t} \int_{V} \rho C_{p} V T=\oint_{A} n_{k} Q_{k} d A+\int_{V} Q^{\prime \prime \prime} d V-\oint_{A} Q_{s} d A
\end{gathered}
$$

In Eq. 1, left-hand side (LHS) terms include transient change and advection of mass, and right-hand side (RHS) terms include source terms for phase change, turbulent mixing, and void drift. In Eq. 2, the first LHS term is transient change in momentum, and there are three terms for momentum advection, which include advection of momentum in the three directions in Cartesian geometry. The RHS terms include 
source terms for gravity, pressure gradient, wall shear, phase change, form loss, and turbulent mixing. Equation 3 LHS terms include transient change in fluid energy and advection of fluid energy, and RHS terms include turbulent mixing, phase change, wall heat transfer, and pressure effects on energy change. Equation 4 includes one term on the LHS for transient change in solid energy, as well as RHS terms for conduction, volumetric energy generation, and heat transfer to the fluid. Symbols in the equations include $\alpha$ for volume fraction, $\rho$ for density, $\vec{V}$ for velocity, and $h$ for enthalpy. Symbol $k$ represents the field, which can be vapor, liquid, and droplets.

Code and solution verification are performed to demonstrate that the code converges to an expected solution and/or at an expected rate. Therefore, efforts are focused on governing equation terms that have an expected convergence rate. For example, one tested term is the momentum equation source term for wall shear. At this point, the particular friction factor model that is employed is not of concern. Rather, the focus is on the fact that the source term in the governing equation will converge to the correct solution at the expected rate based on the equation discretization.

The governing equation terms that must be tested, along with the way they map to intended CTF applications, are summarized in Table 1. The test matrix includes the CTF intended application space in Columns 2-4, which are "Normal PWR operations including core follow," "Departure from nucleate boiling," and "CIPS/CILC." The governing equation terms to be tested are listed in Column 1. These are organized into three main categories: "Solid energy conservation," "Fluid mass and momentum conservation," and "Fluid energy conservation." The important governing equation terms are denoted with an "X" in the appropriate "App space" column. For example, "Grid shear enhancement" is important for CIPS/CILC because of its effects on crud erosion, but it is not significant for normal PWR operating conditions. 


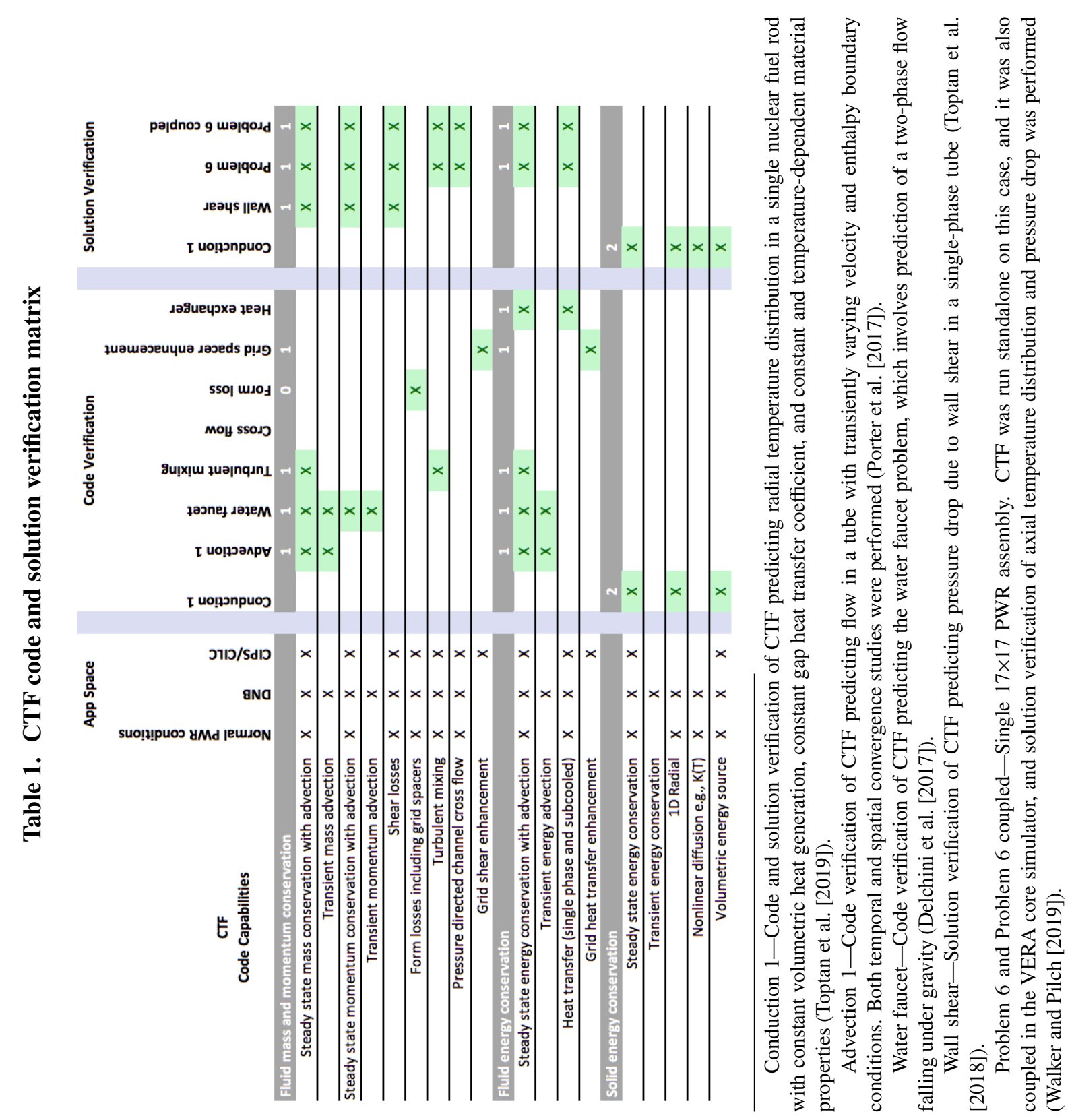


The code verification tests that were performed to verify the CTF capabilities are specified in the center of the table, and the solution verification tests are on the right. Note that the solution verification tests integrate more physics than code verification tests. Each column identifies a separate test that was performed. A number or letter indicates whether the test verifies the code capability listed in that row. For example, the Conduction 1 test demonstrated convergence of the predicted steady-state temperature distribution in a fuel rod to the expected analytical solution when using a constant volumetric heat source and constant gap heat transfer coefficient. An analytical solution of the radial temperature distribution can be calculated for this case, allowing for verification of the radial shape, in addition to the steady-state energy conservation. Furthermore, this test also enabled temperature- and burnup-dependent fuel thermal conductivity models, thus verifying that capability, as well. Because a constant gap conductance was used in this test, the dynamic gap heat transfer model was not verified. Several of these tests were performed before the test matrix shown in Table 1 was generated. These tests are not described in detail in this paper but are instead referenced in the footnotes following the table.

The remainder of the tests - turbulent mixing, cross flow, form loss, grid spacer enhancement, and heat exchanger-were newly developed to fill in gaps of the testing matrix created for this study. The cross flow test does not check off any boxes because work is currently ongoing for this test. It is being developed to verify a code on the directed cross flow modeling capabilities (pressure-driven cross flow) in CTF. The only current gaps in the test matrix are for dynamic gap heat transfer and transient energy convection. The dynamic gap capability consists of a rather complex model that includes the effects of radiative, gas thermal conduction, and contact heat transfer. The thermal conduction and gap closure is also a function of material thermal expansion, burnup-dependent swelling effects, burnup-dependent creep-down, pellet cracking, and pressure driven gap closure. The transient energy conduction will be more straightforward to test and will be addressed in future work. The numbers in the gray bar in the table denote the order of convergence observed for the tests. The fluid solution uses a first-order upwind differencing scheme for the discretization, which accounts for all of the number 1s under fluid mass, energy, and momentum equations. The solid energy conduction uses a central-differencing discretization, which accounts for the number $2 \mathrm{~s}$.

A brief description is provided for each of the newly added code verification tests as part of this study. The following section provides more details on the grid spacer enhancement test as a case study; details on all tests can be found in the CTF Validation and Verification manual (Salko et al. [2019b]).

- Turbulent mixing: A two-channel system with no form losses is set up with identical geometry in each channel so that no pressure-directed cross-flow exists. The inlet enthalpy boundary condition in one channel is increased to create turbulent mixing of energy. An analytical solution is obtained by solving the energy equation for both channels, and the turbulent diffusion model solved by CTF. A mesh refinement is performed to ensure that $\mathrm{CTF}$ converges to the correct solution at the expected first-order rate.

- Cross flow: A model of two different geometry channels is set up with no turbulent mixing or form losses. Inlet boundary conditions are identical. Because of the differences in channel hydraulic diameters, a pressure-driven cross flow force forms due to unequal friction losses in the two different channels. An analytical solution for the outlet flow distribution can be solved by considering the momentum equation in each channel and the friction model employed. Work is ongoing to determine that CTF converges to the correct solution.

- Form loss: A single-channel model with one form loss is created. Fluid properties are constant, and friction and gravitational losses are disabled. The expected pressure loss over the grid can be easily 
calculated and compared to the CTF solution. 



\section{GRID SPACER ENHANCEMENT TEST}

In CTF, the Yao-Hochreiter-Leech (YHL) correlation (Yao et al. [1982]) is used to calculate an exponentially decaying heat transfer and turbulent kinetic energy (TKE) enhancement factor downstream from the spacer grids. The grid spacer enhancement test is a mesh refinement code verification study on the single-phase heat transfer and TKE enhancement due to a single simple (swirl factor set to zero) spacer grid in an axial channel with a heated rod. The expected Nusselt number and TKE enhancement factor obtained by the YHL correlation is tested with the CTF prediction for the rod wall temperature and TKE for first order convergence.

For a single channel with constant specific heat and specified axially uniform heat input, the temperature increase will be linear and is defined as follows:

$$
\dot{m}_{l} C_{p}\left(T_{\text {liq,out }}-T_{\text {liq,in }}\right)=Q_{T} .
$$

The terms $\dot{m}, C_{p}$, and $Q_{T}$ represent the mass flow rate, specific heat capacity at constant pressure, and total heat added to liquid from the heated rod, respectively. For the heated rod, the local wall temperature can be obtained by the solid-liquid interface boundary condition, as follows:

$$
q_{w}^{\prime \prime}=N u_{0} k_{f} \frac{\left(T_{\mathrm{w}, \mathrm{x}}-T_{\mathrm{liq}, \mathrm{x}}\right)}{D_{h}} .
$$

The terms $q_{w}^{\prime \prime}, N u_{0}, k_{f}$, and $D_{h}$ represent the constant wall heat flux, the base Nusselt number, thermal conductivity of the liquid, and hydraulic diameter, respectively. In the current study, the base Nusselt number is set as a constant. The YHL Nusselt number enhancement factor with zero swirl factor is as follows:

$$
E F=1+5.55 \epsilon^{2} \exp \left[-0.13 \frac{\left(x-x_{\text {grid }}\right)}{D_{h}}\right]
$$

The terms $\epsilon, x_{\text {grid }}$, and $E F$ represent the spacer grid blockage, the axial location of the grid spacer, and the grid spacer enhancement factor, respectively. Using a constant friction factor, the wall shear stress can be obtained using the Darcy-Weisbach equation, as follows:

$$
\tau_{w}=\frac{f \rho u^{2}}{8} .
$$

The terms $\tau_{w}$ and $f$ represent the wall shear stress and the friction factor, respectively. Using Eq. 8, the base TKE (upstream from the grid spacer) can be calculated using the relationship discovered by Bradshaw (Salko et al. [2019b]), as follows:

$$
K_{0}=\frac{\tau_{w}}{0.297 \rho} .
$$




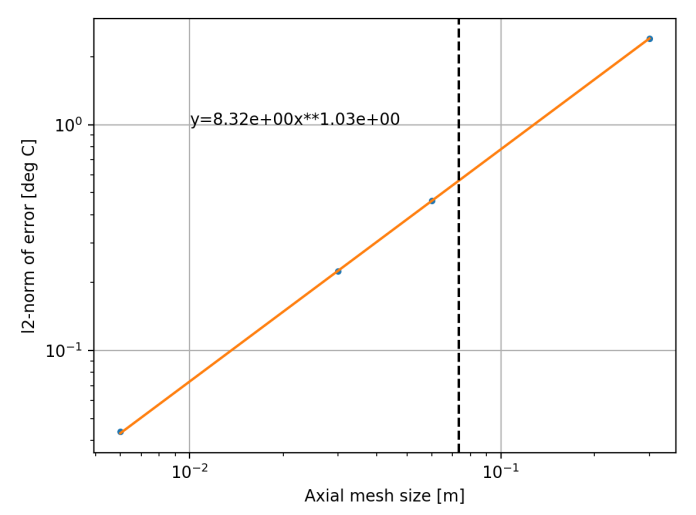

(a) CTF-prediction and curve fit of $L_{2}$ norm of the wall temperature for successive mesh refinements. The dashed vertical line shows the CTF axial mesh size typically used for VERA depletion simulations.

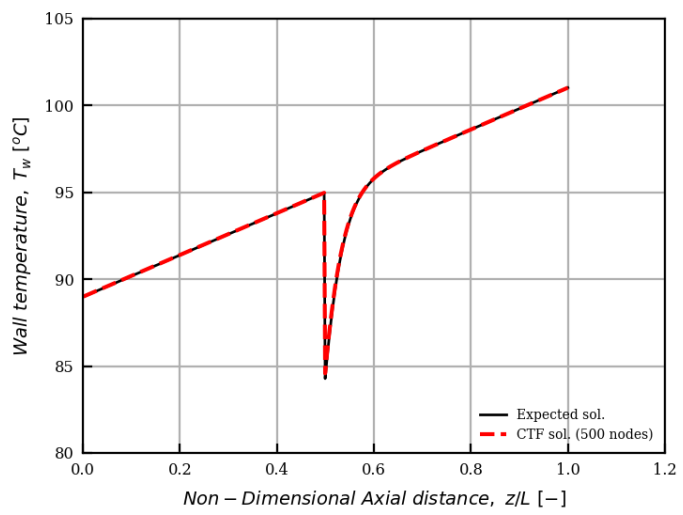

(b) CTF-prediction and expected wall temperature with a spacer grid.

Figure 1. Grid spacer cooling enhancement verification

The term, $K_{0}$ represents the turbulent kinetic energy. The rod surface temperature with grid enhancement effects included is calculated by multiplying the local $E F$ multiplier by $N u_{0}$ in Eq. 6, and the enhanced TKE is obtained by multiplying $E F$ by $K_{0}$ in Eq. 9 .

The model's channel dimensions are set in a manner similar to that used for a PWR subchannel, with a mass source inlet boundary condition of $0.3 \mathrm{~kg} \mathrm{~s}^{-1}$, and an outlet pressure of $155 \mathrm{bar}$, using the following constant thermophysical properties for water: density of $1.000 \mathrm{~kg} \mathrm{~m}^{-3}$, thermal conductivity of $0.65 \mathrm{~W} \mathrm{~m}^{-1} \mathrm{~K}^{-1}$, specific heat capacity of $4.15 \mathrm{~kJ} \mathrm{~kg}^{-1} \mathrm{~K}^{-1}$ and viscosity of $7 \times 10^{-4} \mathrm{~Pa} \mathrm{~s}^{-1}$. The base Nusselt number is set to 50. The spacer grid with a blockage of 0.2 is located at the center of the rod. The friction factor is set to a constant 0.02 . The axial mesh is successively refined with the coarsest mesh having an uniform size of $0.3 \mathrm{~m}$ and the finest mesh with a size of $0.006 \mathrm{~m}$.

Figure 1a shows first order convergence based on the $L_{2}$ norm of the CTF predicted and expected wall temperature for a series of successive mesh refinements. The points represent the error for a CTF simulation performed at a given mesh resolution. The solid line is a curve fit to the data points whose equation is shown on the plot. Finally, a vertical dashed line, which represents the average CTF mesh size $(7.3 \mathrm{~cm})$ used in typical VERA depletion simulations. It can be observed that for this mesh size, the surface temperature numerical error is about $0.56 \mathrm{~K}$. Similarly, Figure $2 \mathrm{~b}$ shows first order convergence based on the $L_{2}$ norm of the CTF predicted and expected TKE for a series of successive mesh refinements. For TKE, the typical mesh size leads to numerical error of roughly $1.3 \times 10^{-3} \mathrm{~J} \mathrm{~kg}^{-1}$. Figure $1 \mathrm{~b}$ shows the comparison between CTF and predicted axial wall temperature profiles. Finally, Figure 2 a shows the comparison between CTF and predicted axial TKE profiles for the finest mesh. The $L_{2}$ norm for the finest mesh for the wall temperature and TKE is on the order of 1e-4 and 1e-5, respectively, demonstrating the good agreement between the CTF prediction and the expected heated wall temperature and TKE.

It is worth noting that the original grid convergence plot that was observed is the one shown in Figure 3. The convergence rate of 0.5 was clearly not expected; this prompted an investigation into the 


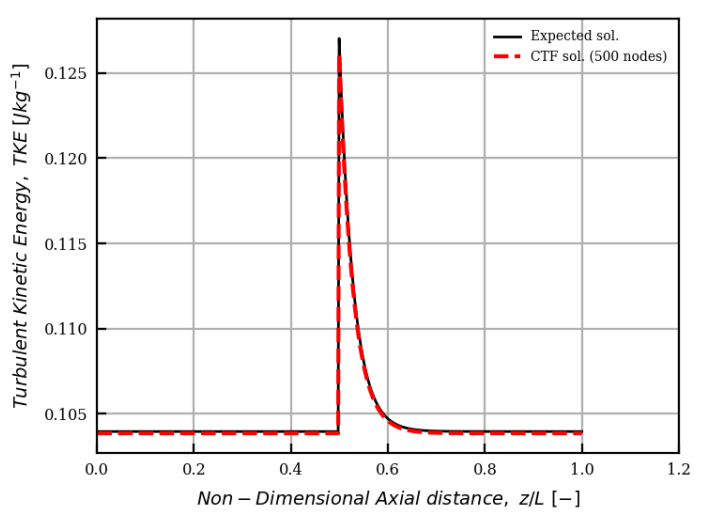

(a) CTF-prediction and expected wall TKE with a spacer grid.

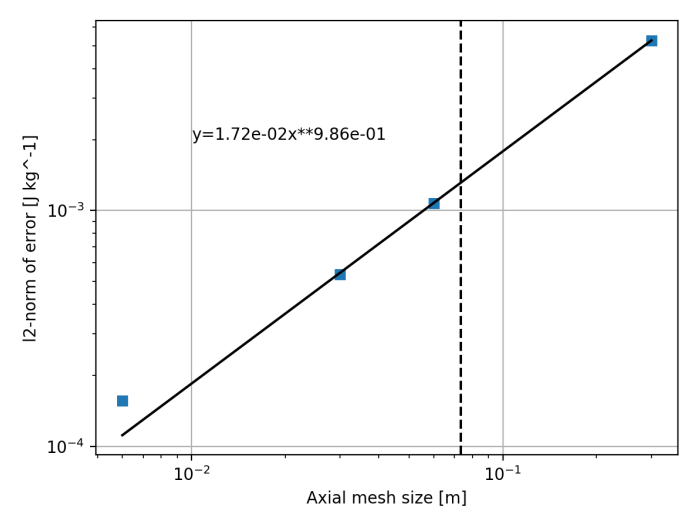

(b) CTF-prediction and curve fit of $L_{2}$ norm of the wall TKE for successive mesh refinements. The dashed vertical line shows the CTF axial mesh size typically used for VERA depletion simulations.

Figure 2. Grid spacer shear enhancement verification

implementation of the TKE enhancement model. This test and investigation revealed a coding defect in which the TKE enhancement was being applied to the incorrect level in the code. This defect was not identified with traditional unit and regression testing. This valuable finding was made possible by development of the code and solution verification testing on this model.

As part of this work, a Python script was developed for automating the process of performing a code/solution verification study. The script does the following:

1. Generates a set of CTF models with successively refined axial meshes from a template input file

2. Runs each case

3. Extracts the results for the selected figure of merit

4. Calculates the analytical solution for the particular problem

5. Calculates the $l_{2}$-norm for each case and generates the code verification plots shown in this report

6. Returns an error if the convergence rate is outside the expected tolerance

This script was modified for each new test that was added to the test matrix so that the analytical solution and figure of merit were applicable to the test of interest. It could also be modified in the future to perform solution verification tests by exchanging the analytical solution for a fine mesh solution. This script is called by the automated CTF test driver, which is run on a daily basis and which posts test results to an archiving system. If any defects are introduced that affect the CTF convergence rate for any added tests, the developer will be notified promptly. 


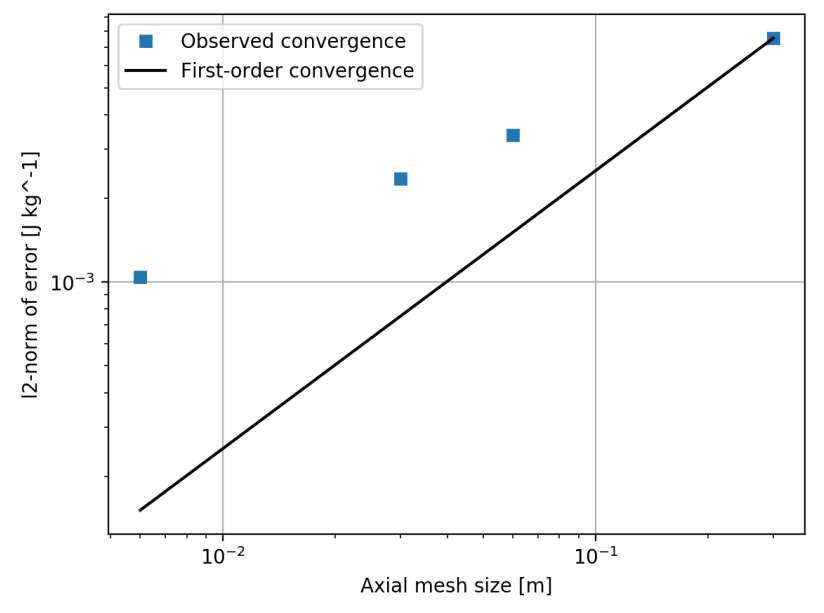

Figure 3. TKE convergence rate with level defect. 


\section{CONCLUSION}

This paper presents an overview of the current state of code and solution verification activities performed on CTF. This test matrix is presented as a table that links intended CTF applications to the required code features for each application and to the individual code and solution verification tests on those features. Development of this matrix led to the identification of gaps in testing. Several new code and solution verification tests were designed and used to test CTF to close the gaps in the testing matrix. In some cases, new code and solution verification testing revealed defects in code features; these defects were fixed as a result of this work. After developing new tests, a Python driver script was developed to automate the testing process so that tests could be re-run quickly and easily. All new tests were added to the automated testing system, which will fail if CTF is not converging to the expected solutions at the expected rate, thus preventing regression of all tested code features. This work resulted in an improvement in CTF SQA, enhancing confidence in code solutions and improving its reliability for intended use cases. 



\section{REFERENCES}

B. Collins, J. Galloway, R. Salko, A. Wysocki, K. Clarno, B. Okhuysen, S. Slattery, N. Adamowicz, D. Andersen, D. Pointer, A. Manera, B. Kendrick, W. Gurecky, V. Petrov, B. Wirth, and J. Rizk. Development of a comprehensive crud-induced power shift (cips) capability within vera. Technical Report CASL-X-2017-1406-000, Consortium for Advanced Simulation of Light Water Reactors, 2017.

Benjamin Collins, Shane Stimpson, Blake W. Kelley, Mitchell T.H. Young, Brendan Kochunas, Aaron Graham, Edward W. Larsen, Thomas Downar, and Andrew Godfrey. Stability and accuracy of 3d neutron transport simulations using the $2 \mathrm{~d} / 1 \mathrm{~d}$ method in mpact. Journal of Computational Physics, 326 : 612 - 628, 2016. ISSN 0021-9991. doi: https://doi.org/10.1016/j.jcp.2016.08.022. URL http://www.sciencedirect.com/science/article/pii/S0021999116303710.

M. Delchini, R. Salko, and V. Mousseau. Implementation of a pressure correction term in the ctf subchannel code. In Transactions of the American Nuclear Society, San Franciso, California, United States of America, 62017.

Brendan Kochunas, Benjamin Collins, Shane Stimpson, Robert Salko, Daniel Jabaay, Aaron Graham, Yuxuan Liu, Kang Seog Kim, William Wieselquist, Andrew Godfrey, Kevin Clarno, Scott Palmtag, Thomas Downar, and Jess Gehin. Vera core simulator methodology for pressurized water reactor cycle depletion. Nuclear Science and Engineering, 185(1):217-231, 2017. doi: 10.13182/NSE16-39. URL https://doi.org/10.13182/NSE16-39.

N. Porter, M. Avramova, and V. Mousseau. Solution verification of ctf and ctf-r using isokinetic advection test problems. In International Conference on Mathematics $\mathcal{E}$ Computational Methods Applied to Nuclear Science \& Engineering, Jeju, Korea, 42017.

U.S. Rohatgi and J.S. Kaizer. Historical perspectives of bepu research in the us. Nuclear Engineering and Design, 358, 2020.

R. Salko, M. Avramova, A. Wysocki, A. Toptan, J. Hu, N. Porter, T. Blyth, C. Dances, A. Gomez, C. Jernigan, J. Kelly, and A. Abarca. CTF Theory Manual. Consortium for Advanced Simulation of Light Water Reactors, 2019a.

R. Salko, A. Wysocki, A. Toptan, N. Porter, T. Blyth, J. Magedanz, C. Dances, M. Gergar, C. Gosdin, C. Jernigan, J. Kelly, V. Kumar, S. Palmtag, J. Gehin, and M. Avramova. CTF Validation and Verification. Consortium for Advanced Simulation of Light Water Reactors, 2019b.

M. Thurgood, J. Kelly, T. Guidotti, R. Kohrt, and K. Crowell. COBRA/TRAC - A Thermal-Hydraulics Code for Transient Analysis of Nuclear Reactor Vessels and Primary Coolant Systems Equations and Constitutive Models. Technical Report NUREG/CR-3046, PNL-4385, Pacific Northwest National Laboratory, 1983.

A. Toptan, N. Porter, R. Salko, and M. Avramova. Implementation and Assessment of Wall Friction Models for LWR Core Analysis. Annals of Nuclear Energy, 115:565-572, 2018.

A. Toptan, R. Salko, M. Avramova, K. Clarno, and D. Kropaczek. A new fuel modeling capability, ctffuel, with a case study on the fuel thermal conductivity degradation. Nuclear Engineering and Design, 341: 248-258, 2019.

C. Unal, B. Williams, F. Hemez, S.H. Atamturktur, and P. McClure. Improved best estimate plus 
uncertainty methodology, including advanced validation concepts, to license evolving nuclear reactors. Nuclear Engineering and Design, 241, 2011.

E. Walker and M. Pilch. Axial solution verification for mpact, ctf th, ctffuel, and vera-cs, 13:phi.v\&v.p19.01. Technical Report CASL-U-2019-1852-000, Consortium for Advanced Simulation of Light Water Reactors, 2019.

S.C. Yao, L.E. Hochreiter, and W.J. Leech. Heat-transfer augmentation in rod bundles near grid spacers. Journal of Heat Transfer, 104:76-81, 1982. 